Monatsschrift f. Geburtshülfe u. Gynäkologie 1931;89:158-160

\title{
Der Zeitschrift für Geburtshilfe zum Gruße
}

Die Zeitschrift für Geburtshilfe und Gynäkologie begeht das Fest des Erscheinens ihres hundertsten Bandes. Die Monatsschrift für Geburtshilfe und Gynäkologie will es sich nicht versagen, der Kollegin in litteris zu diesem Erfolg ihre Glückwünsche und ihre kollegiale Anerkennung darzubringen.

Verfasser ist der letzte noch lebende Zeuge der Entstehung dieser Zeitschrift, der letzte, der seit ihrer Entstehung an ihr mitgearbeitet hat. Er empfindet es als ein officium nobile, seine Erinnerungen aus jener Zeit und aus ihren Entwickhmgsjahren litterarisch festzulegen. Um die Mitte vorigen Jahrhunderts, als die Renaissance der medi-zinischen Wissenschaft, die Virc/iowsche Ära einsetzte, besaß das Fach der Geburtshilfe nur ein eigenes Publikationsorgan, die Monatsschrift für Geburtskunde und Prauenkrankheiten, die damals unter der Leitung von von Riígen, Credê und E. Martin, Hecker und anderen sich bemühte, die Forschungen und Beobachtungen der Fachkollegen zu publizieren. Sie brachte auch Berichte der Verhandlungen der einzigen damals be-stehenden Fachgesellschaft, der geburtshilflichen Gesellschaft in Berlin (gegründet 1845). Die Verhandlungen der geburtshilflichen Sektion der deutschen Versammlungen der Naturforscher und Arzte fanden nur in beschränkter Weise darin einen Widerhall. Weitere lokale und regionale Vereinigungen der Geburtshelfer entwickelten sich erst nach und nach in Leipzig, München und Dresden. Die wissensehaftliche Tätigkeit der Berliner Gesellschaften war eine beschränkte: ihr war nur eine kurze Stunde gewidmet, den Rest des langen Abends füllte das gesellschaft-liche Zusammensein aus. Die Berliner Gesellschaft kam erst zu leb-hafterer Auswertung ihres wissenschaftlichen Materials, als E. Martin 1859 die Berliner Professur übernommen hatte und mit ihm die Reihe seiner ersten Assistentengruppe Olshausen, Gmserow, Winckel,

Der Zeitschrift für Geburtshilfe zum Gruße. 15Q

Frankenhäusser (um nur die ersten zu nennen) - ihre Arbeiten bekannt gaben. Die allgemeine Bewegung, welche sich in jener Zeit auf alien Gebieten der medizinischen Wissenschaft entwickelte, rief naturgemäß den Wunsch nach einer ausgiebigeren Vertretung wach. Il $\alpha$, vornehm-lich diesem Wunsch, entsprang der Entschluß, der die zur Natur for seller-

Versammlung in Salzburg 1872 anwesenden Kollegen bewog, ein leistungsfähigeres, großes modernes Organ zu schaffen. Der geniale Feuerkopf Spíegelberg, Gusserow und eine Reihe anderer beschlossen, eine Zeitschrift zu gründen, an der sie die Gesamtheit der deutschen Vertreter des Faches zu beteiligen in Aussicht nahmen. Sie boten den Eintritt in den Vorstand des von ihnen begründeten Archiv auch den Schrift-leitern der Monatsschrift an. Credé folgte ihrem Ruf, E. Martin lehnte ab. Das ausschlaggebende Motiv zu dieser Ablehnung war die von E. Martin im Verein mit dem Vorstand der Berliner Geburtshilflichen Gesellschaft, welche er damals als Vorsitzender leitete, ihrerseits ge-stellte Bedingung, daß das neue Journal die Verhandlungen der Berliner Gesellschaft in extenso aufnehmen sollte. Diese Forderung wurde, an-geblich auf spezielles Verlangen des gewählten Verlegers Hirschwald in Berlin, abgelehnt. Gewissermaßen als Rückgrat für das Archiv wurde schon damals der Gedanke erwogen, die Gesamtheit der deutschen Geburtshelfer und Gynäkologen zu einer ,, Deutschen Gesellschaft für 
Gynäkologie" zu-sammen zu schließen. Dieser Vorschlag wurde erst durch die geschickte Führung von Wínckel 1887 bei Gelegenheit der Münchener Natur-forscher-Versammlung in die Tat umgesetzt. Auch dann lehnte Hirschwald den Abdruck der ungekürzten Protokolle der neubegründeten Gynäkologischen Gesellschaft ab. Erst als Julius Springer 1921 den Hirschwaldschen Verlag mit dem seinigen vereint hatte, ist das Archiv das offizielle Organ der Deutschen Gesellschaft für Gynäkologie ge-worden.

Die Berliner Geburtshilfliche Gesellschaft spaltete sich 1872 infolge verschiedener Unzukömmlichkeiten. E. Martin trat aus der Gesellschaft aus, seine aktiven Schüler und eine große Zahl seiner sonstigen Anhänger traten zusammen, um eine neue Berliner Gesellschaft für Geburtshilfe und Gynäkologie zu bilden. Sie beschloß sofort, sich auch ein eigenes Organ zu schaffen, die Zeitschrift für Geburtshilfe und Frauenkrankheiten, welche Enke zu drucken übernahm. Die alte Gesellschaft beschloß ihre Tätigkeit fortzusetzen und ihre Arbeiten in „Beiträgen” erscheinen zu lassen, welche zu drucken der Hirschwald-sche Verlag übernahm. E. Martins Ableben 1875 führte die unvermeid-liche Lösung dieses Zustandes herbei. Sein Nachfolger, Carl Schroder, erreichte mühelos die Wiedervereinigung der beiden Gesellschaften, die nunmehr den Namen: ,, Gesellschaft für Geburtshilfe und Gynäkologie zu Berlin” erhielt und die ,, Zeitschrift für Geburtshilfe und Gynäkologie" gründete.

Dieses Publikationsorgan übernahm der En $1 / 8$ esche Verlag, damals in Erlangen. Der erste Band erschien 1877. Schröders geistsprühende Initiative riß nicht nur die von ihm übernommenen derzeitigen Assistenten der Frauenklinik, sondern sehr bald weitgehend Arbeiter unseres Faches mit sich fort. Die junge Zeitschrift fand sehr schnell

160 Dei- Zeitschrift für Geburtshilfe zum Gruße.

einen weiten Treundeskreis. Naeh Schroders jähem, allzufrühem Tode übernahm sein Nachfolger Olshausen die Schriftleitung, um sie bis zu seiner Emeritierung 1910 allein zu führen.

In diesem Zeitabschnitt vollzog sich die überraschende Entwicklung der medizinischen Wissenschaft. Es genügt hier die Namen Koch und Röntgen zu nennen, die Entwicklung der Biologie mit den chemisch-physikalischen Forschungen, um auf die unabsehbar weite Fläche hinzu-weisen, in der sich in fast überstürzender Weise Entdeckungen und Erforschungen folgten, eine Ausgestaltung, über deren Bedeutung für die damalige Zeit, aber auch heute die meisten Mitarbeiter sich kaum noch eine Vorstellung machen können. Die Geburtshilfe und Frauenheilkunde wurde von Erfolg zu Erfolg geführt. Es wurde für jedermann ein dringendes Bedürfnis, zu versuchen, in Fühlung zu bleiben mit den auf alien Einzelgebieten der Medizin erreichten Erfolgen.

Gleichzeitig trat in immer dringlicher Weise das Bedürfnis hervor, das Ergebnis dieser Forschungen in Wort und Bild unmittelbar der Kenntnisnahme und Kritik der Ärztewelt zu unterbreiten. Die Zahl der wissenschaftlichen Vereine wuchs, das Bedürfnis zu mündlichem Aus-tausch führte zu häufigen Tagesfahrten der Vereine.

Staunenswerterweise zeigte sich in alien Kulturländern eine analoge Bewegung. Aus dem Austausch einzelner Fachgenossen aus aller Welt entwickelt sich der Wunsch zu einer internationalen Aussprache. - Diesen Bewegungen gegenüber verhielt sich die Schriftleitung der Zeitschrift für Geburtshilfe und Gynäkologie zurückhaltend, skeptisch, ab-lehnend, Es entstand eine Art Lücke auch in unserer Litteratur, in welche die Monatsschrift für Geburtshilfe und Gynäkologie (A. Martin und N. Saerger) 1894 eintrat. Ihr rascher Erfolg erwies das Vorhandensein des genannten Bedürfnisses. 
Für die Berliner Geburtshilfliche Gesellschaft wurde das Eintreten Bumms zur Erlösung, für die Zeitschrift die Übernahme der Schriftleitung durch Max Hofmeier und Karl Franz. Die Zeitschrift nahm weitergehend Anted an der oben skizzierten wissenschaftlichen Auf-gabe. Die beiden zu voller Tätigkeit ausgerüsteten Berliner Kliniken bildeten die Grundlage für die bedeutungsvollsten Publikationen in der Zeitschrift. Bumms jäher Tod ließ ein breites Arbeitsfeld führerlos zurück!

Das war der Zeitpunkt, in dem W. Stöckel die Führung der Gesell schaft und die Schriftleitung der Zeitschrift übernahm. Seiner organisatorischen Kraft bot sich eine willkommene Aufgabe. Seine wissenschaftliche Tätigkeit, die Fülle der Anregungen, die sich ihm aus seinen wissenschaftlichen Forschungen und Arbeiten ergab, übten ihre Rückwirkung sowohl in der Entwicklung der Geburtshilflichen Gesellschaft als der Zeitschrift aus, so daß sie beide in die Front unseres Faches traten! Möge es Walter Stoeckel vergönnt sein, noch lange Jahre beide zu führen und sich auch an dieser Stelle des vollen Erfolgs seines arbeitsreichen Lebens zu erfreuen! A. Martin.

Personalien.

Dr. Heinz Siedentopf hat sich in Leip*zig für Geburtshilfe und Gynäkologie habilitiert. 